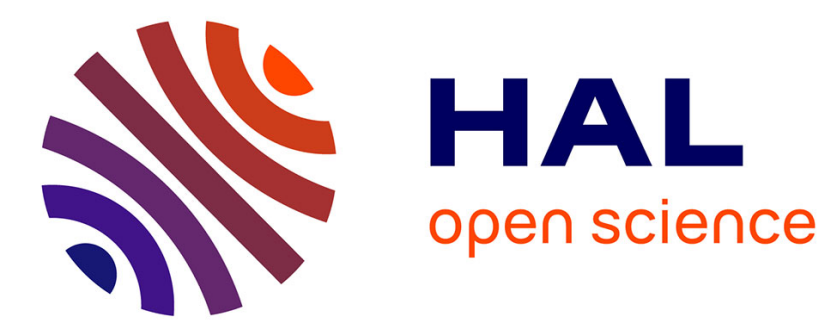

\title{
Deux formules pour devenir adulte en France et en Espagne:
}

\author{
Sandra Gaviria
}

\section{To cite this version:}

Sandra Gaviria. Deux formules pour devenir adulte en France et en Espagne:. Dialogue , 2001, 10.3917/dia.153.0031 . hal-02385913

\section{HAL Id: hal-02385913 \\ https://hal.science/hal-02385913}

Submitted on 29 Nov 2019

HAL is a multi-disciplinary open access archive for the deposit and dissemination of scientific research documents, whether they are published or not. The documents may come from teaching and research institutions in France or abroad, or from public or private research centers.
L'archive ouverte pluridisciplinaire HAL, est destinée au dépôt et à la diffusion de documents scientifiques de niveau recherche, publiés ou non, émanant des établissements d'enseignement et de recherche français ou étrangers, des laboratoires publics ou privés. 
chercher : repérer : avancer

Cet article est disponible en ligne à l'adresse :

http://www.cairn.info/article.php?ID REVUE=DIA\&ID NUMPUBLIE=DIA_153\&ID_ARTICLE=DIA 1530031

Deux formules pour devenir adulte : en France et en Espagne

\author{
par Sandra GAVIRIA
}

\title{
érès | Dialogue
}

$2001 / 3-n^{\circ} 153$

ISSN 0242-8962 | ISBN 2-8658-6905-9 | pages 31 à 39

Pour citer cet article :

- Gaviria S., Deux formules pour devenir adulte : en France et en Espagne, Dialogue 2001/ 3, n 153, p. 31-39.

Distribution électronique Cairn pour érès.

(C) érès. Tous droits réservés pour tous pays.

La reproduction ou représentation de cet article, notamment par photocopie, n'est autorisée que dans les limites des conditions générales d'utilisation du site ou, le cas échéant, des conditions générales de la licence souscrite par votre établissement. Toute autre reproduction ou représentation, en tout ou partie, sous quelque forme et de quelque manière que ce soit, est interdite sauf accord préalable et écrit de l'éditeur, en dehors des cas prévus par la législation en vigueur en France. Il est précisé que son stockage dans une base de données est également interdit. 


\section{Deux formules pour devenir adulte : en France et en Espagne}

SANDRA GAVIRIA

Ana est Espagnole. Vingt-sept ans, célibataire, ingénieur, elle vit chez ses parents. Cela lui semble naturel: «Je ne veux pas partir seule. Je ne veux pas partir non plus pour être dans de plus mauvaises conditions que chez mes parents ; chez mes parents, je suis très bien, je suis très contente, en réalité, j'ai tout. »

Jean-Luc, lui, est Français. Vingt-six ans, célibataire, professeur de sport : il vit seul. Il nous dit : «Un jeune qui vit avec ses parents, ça a trop tendance à créer peut-être une copie conforme d'eux, donc... Je pense que les relations avec ses parents, s'ils sont trop proches, créent une perte d'autonomie évidente, une perte de liberté, en tout cas sur le plan intellectuel. Le fait de se rattacher trop longtemps à son père ou à sa mère, pour moi, c'est surtout... la perte d'autonomie. »

Ces propos ne sont pas le fruit du hasard, ou de la seule psychologie d'Ana et de Jean-Luc. Ils traduisent deux façons différentes d'entrer dans la vie adulte : les jeunes Français quittent leurs parents bien avant leurs voisins du Sud, lesquels ont du mal à laisser le nid vide. Mais comment expliquer cette différence ? Les jeunes Français sont-ils moins attachés à leurs parents ? Ont-ils plus d'argent ? L'objet de cet article est de montrer que les trajectoires des jeunes Français et des jeunes Espagnols diffèrent parce que des deux côtés des Pyrénées les conceptions divergent quant à la marche à suivre pour 
devenir un individu adulte indépendant, autonome et épanoui. En France, certains auteurs considèrent qu'un jeune n'est adulte que lorsqu'il n'habite plus chez ses parents et qu'il travaille (Galland, 1993). Il en va autrement en Espagne, où être considéré comme adulte n'est pas aussi fortement lié au départ du domicile parental.

\section{Le moment de partir}

En France, l'apprentissage de l'autonomie est considéré comme un processus constitutif de l'accès à l'âge adulte. Les parents en font un objectif éducatif majeur (Singly 1996). En un sens, leur principal rôle est de favoriser le départ des enfants (Maunaye, 1998). Le départ du domicile parental est donc jugé comme une étape nécessaire dans la construction de la personnalité. De leur côté, les jeunes considèrent que rester trop longtemps chez leurs parents constitue un handicap : comme leurs parents, ils soulignent l'importance que revêt à leurs yeux de "couper le cordon », d'avoir un espace qui leur soit propre et de gérer leur temps et leur vie sans la contrainte parentale.

En Espagne, il n'en est pas de même. Les parents défendent l'idée que l'enfant ne peut devenir un adulte épanoui que s'il a un fort sentiment d'appartenance familiale. À leurs yeux, tant que les enfants ne sont pas mariés, le foyer parental est le lieu qui convient le mieux à leur équilibre. Le bon parent est celui qui crée un foyer agréable pour ses enfants et qui accepte qu'ils y restent le temps qu'ils veulent. Ce sont surtout les mères de famille qui s'occupent de créer l'ambiance familiale propice à l'épanouissement de leur enfant. Bien souvent, elles ne sont pas actives (en 1994, seules $30 \%$ des femmes de 45 ans étaient actives ${ }^{1}$ ) et elles se consacrent en priorité à leurs enfants. Leurs plaisirs personnels passent après ceux des membres du foyer. L'étude sur la famille en Espagne d'Ines Alberdi (1994) montre que, pour $75 \%$ des personnes interrogées, « le devoir des parents est d'essayer de faire au mieux pour leurs enfants, même s'ils doivent sacrifier leur propre bon heur».

C'est ainsi que, pour des parents espagnols, le fait que leur fils ou leur fille décide de prendre un appartement dans la même ville qu'eux pour y habiter seul est synonyme d'échec. Ils se sentent déshonorés et suspects : ils savent que leur entourage ne manquera pas de s'interroger sur leur compétence parentale. De leur côté, la plupart des jeunes disent se sentir bien dans le foyer familial et ne pas vouloir le quitter avant le mariage. Ils mettent en avant le fait qu'ils n'aiment pas la solitude et que leurs parents seraient tristes et frustrés s'ils partaient. Ils comprennent mal pourquoi, en France, on considère comme anormal qu'un jeune qui travaille et a un bon salaire reste chez ses parents tant qu'il n'est pas marié. 


\section{L'envol du nid}

En 1995, en France, seulement $17 \%$ des jeunes de 25 à 29 ans vivaient chez leurs parents ; en Espagne, ils étaient $59 \%$ (Eurostat 1997). La proportion n'est donc pas la même. Les circonstances du départ et la trajectoire des jeunes varient également d'un pays à l'autre. Les jeunes Français quittent habituellement le foyer familial longtemps avant leur mariage. Ensuite alternent des périodes où ils habitent seuls, des périodes de vie en concubinage et, parfois, surtout en cas de rupture, des périodes où ils retournent vivre chez leurs parents (Blöss, 1990). En revanche, 70\% des jeunes Espagnols ne partent de chez leurs parents qu'au moment de leur mariage (Leal, 1997).

Les conditions matérielles qui entourent le départ des jeunes dans les deux pays semblent être différentes. En France, leur premier logement indépendant est souvent loué et aménagé de meubles de faible valeur, achetés d'occasion ou récupérés dans la famille. Il est courant que le jeune reçoive dans les premiers temps de l'aide financière des parents, soit parce qu'il est encore étudiant, soit parce que sa situation professionnelle reste empreinte de précarité.

On peut dire qu'en France, le départ du domicile parental constitue une étape importante au sein d'un processus d'autonomisation qui n'est pas encore achevé et qui prend du temps. Il n'en est pas de même en Espagne, où le départ des jeunes n'intervient que lorsqu'ils ont acquis une stabilité professionnelle et donc économique (Garrido, 1997). Pour eux, le départ et le mariage marquent la conjonction d'un ensemble de conditions favorables. Le cas typique est le suivant : le jeune travaille depuis quelques années et il a eu le temps d'économiser de l'argent sur un compte épargne-logement. Avant le mariage, il achète avec son partenaire un logement. Parfois, les parents aident à l'achat, mais cela ne dispense pas les jeunes conjoints de contracter un emprunt bancaire remboursable sur dix ou quinze ans. Cette volonté d'accéder à la propriété n'est pas spécifique à la jeunesse. Elle se retrouve dans l'ensemble de la population : en Espagne, $95 \%$ des familles sont propriétaires de leur logement (Leal, 1997), contre seulement $65 \%$ en France.

\section{Être un jeune adulte en France et en Espagne}

L'analyse des attitudes des jeunes de 25 à 29 ans des deux pays à l'égard du domicile parental nous conduit à interpréter leurs divergences comme la traduction de deux conceptions différentes de la jeunesse. L'âge au départ ne serait qu'une manifestation particulièrement apparente et aisément quantifiable de la différence de deux modes de vie, de deux manières de se construire et de deux normes de sociabilité ${ }^{2}$. 


\section{La responsabilisation financière}

L'analyse des rapports d'argent entre parents et enfants des deux côtés des Pyrénées montre qu'en France l'éducation par la responsabilisation des jeunes est plus poussée qu'en Espagne.

Les jeunes Français, qu'ils soient étudiants ou en début de carrière professionnelle, sont souvent « ric-rac » économiquement. Il est courant que l'étudiant travaille pour se payer des vacances ou arrondir ses fins de mois. Le jeune qui travaille, lui, doit consacrer la majeure partie de son salaire à faire face au coût de la vie quotidienne : loyer, factures, nourriture, il lui reste peu d'argent pour ses loisirs. Tania, 22 ans, étudiante et employée, explique les avantages et les inconvénients de la vie hors du domicile parental : «Je ne gagne pas beaucoup, j'ai des contraintes, je dois payer un appartement etc., mais je vis. Si je n'ai pas envie de ranger ma chambre, je ne la range pas, c'est bête ce que je dis, mais... si je n'ai pas envie de mettre mes chaus sons, je ne les mets pas. Voilà. Ce sont des petits trucs qui nous pourrissent la vie quotidienne lorsqu'on est chez ses parents. »

La plus grande responsabilisation des jeunes Français est pour partie un effet mécanique de leur départ précoce. Cependant, même quand ils vivent encore chez leurs parents, la tendance est la même : ces derniers, quel que soit leur niveau de vie, insistent plus qu'en Espagne pour que le jeune travaille l'été afin d'assumer une part de ses frais personnels et de prendre conscience de l'effort nécessaire pour gagner sa vie. Et, lorsque le jeune a un emploi, il est courant que les parents lui demandent une participation financière plus ou moins symbolique.

Résider longtemps chez leurs parents permet aux jeunes Espagnols d'avoir moins de soucis financiers. Lorsqu'ils font des études, si leurs parents ont les moyens de subvenir à leurs besoins, ces derniers ne les contraignent pas à faire une incursion dans le monde du travail. Si les parents ont des moyens plus réduits, les jeunes travaillent pendant l'été, mais ils sont peu nombreux à travailler pendant l'année universitaire. Et, lorsqu'ils travaillent, ils ne participent aux frais du foyer que dans les milieux sociaux les plus défavorisés, lorsque les parents ne peuvent se passer de cette aide financière. Les jeunes qui ont un emploi et dont les parents n'ont pas de difficultés économiques majeures gardent la totalité de leur salaire pour leurs dépenses personnelles, voiture, vêtements, sorties, ou bien économisent pour leur futur logement. C'est le cas de Lázaro, 30 ans, architecte, qui décrit un système familial dans lequel sa participation serait presque incongrue : "Chez moi, je n'apporte pas d'argent, je n'aide pas mes parents, car ils ne veulent pas et me disent d'économiser. » Ainsi, à l'instar de ses pairs, il n'a guère de soucis financiers, ce qui n'est pas le cas des jeunes Français.

La force de ce modèle ressort clairement du discours de Noelia qui, à 25 ans, est coiffeuse. Contrairement à la majorité des parents, les siens, alors qu'ils n'en ont pas besoin, exigent qu'elle leur verse une partie de son salaire. Elle dit : "Le jour où je ne donnerai plus une partie de mon salaire à mes 
parents, je devrai partir. Pour moi, c'est bizarre, non seulement bizarre mais anormal, parce que ce qui est normal est que tes parents t'aident et qu'ils t'appuient. »

\section{Les relations entre parents et enfants}

Dans les deux pays, les rapports entre parents et enfants sont jugés bons (Galland, 1996 ; Alberdi, 1994). Les règles qui régissaient les rapports familiaux traditionnels semblent s'être assouplies. Comme l'explique François de Singly (1993), la famille contemporaine n'est plus un lieu où règne le pouvoir autoritaire du père : il y règne aujourd'hui « une nouvelle exigence dans le domaine des relations, qui doivent être vécues sur le mode non de la contrainte, mais de l'élection ». Le sociologue Lluis Flaquer (1998), de son côté, montre que la famille espagnole est régie par la négociation. Néanmoins, les relations au sein des familles ne se tissent pas de la même façon dans les deux pays.

En France, la précocité de l'installation du jeune dans un logement séparé entraîne une plus grande indépendance entre parents et enfants que ce qu'on voit en Espagne. Les rencontres permettent le maintien de liens affectifs, mais chacun choisit ce qu'il veut protéger du regard des autres. Ces rencontres ne sont que rarement le fruit d'une improvisation. Habituellement, les jeunes préviennent leurs parents avant de leur rendre visite, de venir manger ou de rester dormir. À leurs yeux, la maison des parents n'est plus la leur. De même, les parents ne semblent pas aller très souvent chez leurs enfants et, lorsqu'ils le font, c'est normalement à la suite d'une invitation ou d'un rendez-vous. Ainsi se définissent des territoires personnels et s'ancre le respect d'une intimité non partagée. Bien sûr, le maintien de cette distance n'est pas l'apanage des rapports de filiation. De façon plus générale, ils semblent correspondre aux codes de conduite de la société française.

Les parents français ne connaissent donc le monde dans lequel évoluent leurs enfants que de façon indirecte : ils se le représentent surtout à travers les discussions qu'ils ont avec ces derniers. En pratique, ils ne connaissent pas bien leur entourage et leur mode de vie. Cela ne semble poser problème ni aux uns ni aux autres, et les jeunes n'expriment pas le désir de voir se croiser leurs différentes sphères de sociabilité. Au contraire, ils semblent penser qu'il est préférable que les sphères restent séparées.

En Espagne, la vie des jeunes et celle des parents se croisent plus souvent, notamment parce qu'ils résident plus longtemps dans le même logement. Ni les jeunes ni les parents ne voient d'inconvénients, bien au contraire, à ce que leurs vies s'entremêlent. Ainsi, les parents connaissent très bien les amis de leurs enfants. Ils savent qui sont leurs parents, ce qu'ils font, qui est la copine ou le copain de chacun, les études qu'ils suivent ou l'activité professionnelle qu'ils ont. Il est courant que les différentes générations aient des activités ensemble, organisent, par exemple, un repas entre parents, enfants et amis des uns ou des autres. En été, les familles (grands-parents, 
oncles et tantes) et les jeunes se réunissent souvent, les résidences secondaires sont chose courante et elles deviennent un lieu de rencontre durant l'été.

Il semble en fait qu'en Espagne ce qu'on appelle le territoire intime ait un périmètre plus restreint qu'en France. En effet, même lorsque les enfants sont mariés, les règles qui régissent leurs rapports avec leurs parents et leur accès à la maison familiale sont différentes de ce qu'on voit en France. Par exemple, il n'est pas rare que les jeunes aient la clé de leurs parents et entrent chez ces derniers sans sonner ni prévenir. Ils continuent de considérer la maison de leurs parents comme la leur.

\section{La vie de couple}

Les jeunes des deux pays se mettent en couple à des moments différents de leur cycle de vie et dans des circonstances dissemblables. En France, il est courant de voir des jeunes résider en couple alors qu'ils n'ont pas une complète indépendance économique, tandis qu'en Espagne l'installation conjugale, normalement synonyme de mariage, est impensable avant une totale indépendance financière.

La majorité des jeunes Français vivent en concubinage plusieurs années avant de se marier ou d'avoir une stabilité professionnelle. Pour eux, vivre à deux est une étape nécessaire avant d'envisager le mariage. Jeanne, 30 ans, qui vit en concubinage, justifie ainsi son choix : «Tu connais la personne en vivant avec elle. Tu la connais mieux que si tu sors avec elle, si tu passes juste des petites vacances etc. Ça n'a rien à voir réellement jour après jour. Tu connais les défauts de l'autre. » La cohabitation est elle-même souvent progressive. L'installation ne fait qu'entériner une réalité déjà existante. Au fur et à mesure de l'évolution de leur relation, le jeune homme va de plus en plus souvent chez la jeune femme et y reste de plus en plus longtemps - ou inversement -, et ce jusqu'à ce que la décision de cohabiter devienne une quasiévidence (Kaufmann, 1992). Il est également courant qu'avant de se marier un jeune ait eu plus d'une expérience de concubinage. Le mariage n'intervient souvent que lorsque le couple désire avoir des enfants (Leridon, 1988).

Pour ces jeunes, le concubinage n'implique pas forcément l'intégration du partenaire dans sa famille lors d'événements tels que les fêtes de Noël ou les visites chez les parents. De même, l'argent reste une affaire individuelle. Une caisse commune se crée pour le règlement des factures communes et l'achat de nourriture, mais la propriété des objets et des appareils ménagers reste distincte. En début de relation, il n'y a que peu d'achats d'objets en commun. Les jeunes semblent anticiper la possibilité d'une rupture. Ils évitent donc la communauté qui induirait un problème de partage.

Les jeunes Espagnols n'ont pas le même itinéraire. Ils connaissent souvent de longues fiançailles ${ }^{3}$, car ils se marient uniquement quand toutes les conditions d'une grande stabilité sont réunies. Les couples se marient en général quatre ou cinq ans après le début de leur relation, années durant les- 
quelles chacun habite chez ses parents. Marta, fiancée depuis cinq ans et résidant chez sa mère, explique sa préférence pour le mariage en réfutant les avantages que l'on prête au concubinage : "D'accord, le concubinage a des avantages, tu commences à te connaître, à connaître les défauts de l'autre avant qu'il ne soit trop tard. Mais, finalement, je crois que, si tu prends une option, il faut au moins l'essayer jusqu'au bout, et ce n'est pas pareil d'être avec quelqu'un quand tu sais que si ça ne marche pas tu peux arrêter. Quand tu es marié, ce n'est pas aussi facile de se séparer. »

Durant leur période de fiançailles, les jeunes fonctionnent comme un couple, sauf qu'ils n'habitent pas ensemble. Souvent, ils sont complètement intégrés dans la famille du partenaire, la seule restriction - communément admise en Espagne - étant de ne pas coucher dans le même lit quand on est chez les parents. Cette norme largement partagée reste le plus souvent implicite, les jeunes n'exprimant généralement pas le souhait de dormir ensemble chez leurs parents. À leur sens, cela constituerait un manque de respect envers leurs parents, ils disent qu'ils n'oseraient pas le faire même si ces derniers l'acceptaient. Pour avoir des relations sexuelles, les jeunes trouvent diverses solutions comme la voiture, le départ en week-end ou l'appartement d'amis.

Bien sûr, tous les jeunes Espagnols ne suivent pas la même trajectoire. Certains choisissent le concubinage. Les chiffres sont cependant parlants. En 1995, les jeunes de 25 à 29 ans qui optent pour le concubinage ne représentent que $4 \%$ (Eurostat, 1997). Bien que certains spécialistes comme Lluis Flaquer (1998) estiment qu'ils sont en réalité au moins $7 \%$, le phénomène n'est pas aussi répandu qu'en France, où $25 \%$ de jeunes de ces tranches d'âges vivent en concubinage. Ces jeunes couples ont en outre des caractéristiques différentes puisque, en Espagne, la décision de la cohabitation intervient en amont de l'installation. Elle n'entérine pas un état de fait.

\section{Les amis}

En Espagne, quelle que soit la tranche d'âge, la vie sociale est très intense. Passer à l'improviste chez quelqu'un, par exemple, n'est pas considéré comme une intrusion dans sa vie privée. Au contraire, la visite impromptue est une preuve d'affection. Il est également courant que les gens conservent leurs amis d'enfance ou de jeunesse tout au long de leur vie. Les relations d'amitié sont de la sorte fréquentes et stables. Cette intense sociabilité est favorisée par une faible mobilité géographique. Bien souvent, les jeunes préfèrent perdre leur emploi et se retrouver au chômage plutôt que d'accepter une mutation géographique qui les éloignerait de leur entourage. Pour les jeunes Espagnols, la sociabilité amicale est essentielle à leur équilibre. Être en couple ne signifie pas la restriction de leur vie sociale. Normalement, les couples sortent fréquemment avec le groupe d'amis de l'un des membres et de temps en temps avec celui de l'autre. De même, les sorties 
entre filles ou garçons, qui leur permettent de revenir en quelque sorte au statut de « célibataire », sont monnaie courante.

En France, le fait d'être ou ne pas être en couple conditionne largement le type de sociabilité. Les jeunes célibataires ont une vie sociale intense et les amis sont pour eux une référence identitaire importante. Une fois en couple, les jeunes réduisent énormément leur cercle amical et leur vie sociale. Ils fréquentent en outre de moins en moins de célibataires et de plus en plus de couples. Souvent, ils conservent quelques bons amis, mais ceux-ci ne forment pas un groupe.

\section{Se construire pour devenir adulte}

Les divergences récurrentes que l'on observe entre le cheminement des jeunes Français et celui des jeunes Espagnols vers l'autonomie s'inscrivent dans des logiques distinctes. Les jeunes Français suivent une logique d'expérimentation, et les jeunes Espagnols s'inscrivent dans une logique de stabilité. En effet, lorsqu'ils quittent leurs parents, les Français ne sont qu'au début d'un long parcours incertain. Ils vivront peut-être en concubinage pendant quelques années, puis se retrouveront seuls, ou retourneront parfois pour une courte période vivre chez leurs parents avant de repartir... Les jeunes Espagnols, au contraire, ne partent de chez leurs parents que définitivement, lorsqu'ils sont sûrs de retrouver la stabilité et le confort dont ils jouissaient jusque là. Ils vivent alors entourés, ont un emploi stable et possèdent un appartement correctement aménagé.

En France, pour un jeune, se construire en tant qu'adulte signifie se détacher de son statut d'enfant pour développer de plus en plus son identité personnelle et se construire en tant qu'individu. Parallèlement à ce processus, nous avons observé que les jeunes passent très tôt au statut de "partenaire de » et relèguent au deuxième plan leur identité filiale. Du fait de la séparation des territoires intimes entre parents et enfants, les jeunes Français développent en effet leur identité filiale et leur identité conjugale dans des moments et des espaces différents. Nous pensons qu'ils sont amenés à développer une identité clivée.

En Espagne, les jeunes vivent au sein de sphères de sociabilité intimement imbriquées. Ils se voient ainsi contraints de trouver une unité de soi où pourront fusionner leurs différents statuts, celui de « partenaire de », celui « d'ami de » et de celui de « fils ou fille de ». Leur identité personnelle se développe de façon plus encadrée.

Sandra Gaviria Université Paris 5,

Centre de recherche sur les liens sociaux (CERLIS), 45, rue des Saints-Pères, 75270 Paris cedex 06 


\section{BIBLIOGRAPHIE}

AlBERDI, I. 1994. Informe sobre la situación de la familia en España, Madrid, Ministerio de Trabajo y Asuntos Sociales.

BlÖSS, A. ; FRICKEY, F. ; GODARD, F. 1990. « Cohabiter, décohabiter, recohabiter. Itinéraires de deux générations de femmes », Revue française de sociologie, ${ }^{\circ} 31$, p. 553-572.

Centro de investigaciones sociológicas, juin 1997, Datos de opinión.

EuRostat. 1997. Les Jeunes de l'Union européenne, Communautés européennes.

Flaquer, L. 1998. El destino de la familia, Madrid, Ariel.

GALlAND, O. 1993. «La jeunesse en France, un nouvel âge de la vie », dans Cavalli A., Galland O. (sous la direction de), L'Allongement de la jeunesse, Arles, Actes Sud, p. 19-40.

Galland, O. 1996. Les Étudiants, Paris, La Découverte.

Garrido, L. ; Requena, M. 1997. La emancipación de los jóvenes en España, Madrid, Ed Ministerio de Trabajo y Asuntos Sociales.

GAVIRIA, S. 1998. Les Jeunes en France et en Espagne : vivre ou ne pas vivre au domicile parental ? Mémoire de DEA (sous la direction de F. de Singly), université Paris 5.

Kaufmann, J.-C. 1992. La Trame conjugale. Analyse du couple par son linge, Paris, Nathan. LEAL, J. 1997. La edad de emancipación de los jóvenes, Barcelona, Ricard Vergés.

Leridon, H. ; Villeneuve-GoKalP, C. 1988. «Les nouveaux couples », Population, n 2 , p. 331-374.

Mauger, G. ; Fossé-PoliaK, C. 1992. « De la cohabitation chez les jeunes de milieux populaires (1975-1985) », Dialogue, recherches cliniques et sociologiques sur le couple et la famille, $\mathrm{n} 92$ (De l'union libre), p. 76-87.

Maunaye, E. 1998. Le Départ des enfants, thèse de doctorat (sous la direction de F. de Singly), université Paris 5-Sorbonne.

SingLY, F. de. 1993. Sociologie de la famille contemporaine, Paris, Nathan.

Singly, F. de. 1996. Le Soi, le couple et la famille, Paris, Nathan.

\section{RÉSUMÉ}

Les jeunes Français et les jeunes Espagnols quittent le domicile parental à des moments différents de leur cycle de vie : les premiers partent avant les seconds. Cet écart chiffrable traduit des pratiques et des logiques divergentes. Les jeunes des deux pays ne construisent pas leur identité de la même manière. En France, l'apprentissage de l'autonomie est considéré comme un processus constitutif de l'accès à l'âge adulte, tandis qu'en Espagne l'accent est mis sur l'acquisition d'un fort sentiment d'appartenance familiale. Cette différence de normes nous conduit finalement à penser que les jeunes Français se construisent à partir d'une logique d'expérimentation, alors que les jeunes Espagnols privilégient une logique de stabilité.

\section{Mots CLÉS}

Jeunes. Parents. Domicile. Âge adulte. Autonomisation. Construction de l'identité. France, Espagne.

\section{NoTES}

1. Centro de investigaciones sociológicas, junio 1997, Datos de opinión.

2. Centré sur la comparaison des normes françaises et espagnoles, l'article passe sous silence les comportements minoritaires communs aux deux sociétés mais minoritaires dans chacune d'elles.

3. Les membres des couples stables sont considérés comme étant fiancés (novios), même si la cérémonie des fiançailles ne se fait qu'un ou deux mois avant le mariage. 